J3eA, Journal sur l'enseignement des sciences et technologies de l'information et des systèmes,

Volume 3, Hors-Série 1, 8 (2004)

DOI : http://dx.doi.org/10.1051/bib-j3ea:2004608

(C) EDP Sciences, 2004

De l'utilité de la couleur en localisation et navigation : application à un robot d'assistance

C. Montagne ${ }^{1}$, S. Lelandais ${ }^{1}$ et J. Triboulet ${ }^{2}$

${ }^{1}$ Laboratoire des Systèmes Complexes (LSC)

Université d'Évry

40 rue du Pelvoux

F-91020 Évry CEDEX, France

http://lsc.cemif.univ-evry.fr:8080/

${ }^{2}$ Centre d'Électronique et de Micro-optoélectronique de Montpellier (CEM2)

Université de Montpellier II

Place Gabriel Péri

F-30021 Nîmes CEDEX 01, France

cmontagne@iup.univ-evry.fr 


\title{
De l'utilité de la couleur en localisation et navigation : Application à un robot d'assistance
}

\author{
Christophe Montagne ${ }^{1}$, Sylvie Lelandais ${ }^{1}$, Jean Triboulet ${ }^{2}$ \\ ${ }^{1}$ Laboratoire Systèmes Complexes (LSC), Université d'Evry \\ 40, rue du Pelvoux - 91020 Evry Cedex \\ Site Internet : http://1sc.cemif.univ-evry.fr:8080/ \\ ${ }^{2}$ Centre d'Electronique et de Micro-optoélectronique de Montpellier (CEM2) \\ Université de Montpellier 2 \\ Place Gabriel Péri - 30021 Nîmes Cedex 01 \\ cmontagne@iup.univ-evry.fr
}

\begin{abstract}
Résumé - Dans le cadre d'un projet d'assistance aux personnes handicapées sur leur lieu de vie, notre laboratoire développe une base mobile semi-autonome. L'environnement domestique dans lequel évolue cette base est riche en couleurs et en textures. De plus, les objets qu'on y trouve sont le plus souvent structurés et donc assimilables à des formes géométriques simples. Nous souhaitons utiliser la couleur pour la localisation et la navigation d'un robot. Notre travail se fonde sur une méthode opérationnelle basée sur l'appariement de segments de droite extraits d'images monochromatiques. Nous voulons aussi évaluer l'apport de l'utilisation de la couleur par rapport aux niveaux de gris. La problématique qui se pose tient au choix d'un espace de représentation des couleurs et au choix d'une méthode d'extraction des segments tenant compte de la couleur.
\end{abstract}

Mots Clé : Environnement domestique, évaluation d'espace couleur, extraction de segments, vision pour la robotique.

\section{INTRODUCTION}

Les activités scientifiques du LSC concernent la problématique dite de la «machine intelligente». Les travaux, ainsi menés, tendent à la réalisation de machines ou systèmes physiques en mesure de percevoir et d'agir dans un environnement variable (connu ou inconnu), capables de raisonner de façon rationnelle sur une diversité de tâches ou de données et sur les moyens nécessaires pour les accomplir de façon autonome ou en coopération avec un opérateur humain. Cette optique de recherche englobe par conséquent de nombreux domaines dont le traitement des données, la réalité virtuelle, la vision artificielle, la modélisation et le contrôle des systèmes complexes.

Historiquement, le LSC a étendu ses activités au génie biologique et médical au travers de projets ponctuels. Mais, suite à la thèse de Philippe Hoppenot sur la « contribution de la robotique mobile à l'assistance aux personnes handicapées " [1] en collaboration avec l'Association Française contre les Myopathies (AFM), cette activité, jusque là marginale, a pris de l'ampleur et des partenariats se sont noués entre le LSC et d'autres équipes de recherche, des associations de particuliers et des organismes travaillant auprès des personnes handicapées.

Actuellement, un projet d'Assistance Robotique aux Personnes Handicapées (ARPH) [2] est mené en collaboration avec l'AFM. L'objectif de ce projet est de proposer un appareil d'assistance n'excluant pas la personne handicapée. La philosophie de ARPH est que le robot ne doit pas «faire à la place de » mais compenser les déficiences de la personne. Par conséquent les deux entités deviennent dépendantes et une coopération homme-machine se révèle nécessaire. Le robot développé consiste en une base mobile autonome à laquelle est adjoint un bras manipulateur destiné à être une extension de la personne handicapée Le bras permet ainsi d'effectuer seul différentes tâches de la vie courante tels qu'ouvrir une porte ou verser de l'eau dans un verre. Par ailleurs, la personne handicapée n'ayant pas nécessairement des moyens financiers étendus, le coût du robot doit être limité. Cela induit l'utilisation de capteurs tels l'odométrie et des capteurs à ultrasons de qualité moyenne. Une caméra stéréo équipe toutefois le robot et permet le retour visuel à l'opérateur.

La base en elle-même existe et les travaux actuellement menés concernent la coopération entre l'homme et la machine qui ont tous deux des capacités propres de perception, de décision et d'action. Différentes possibilités de coopération sont envisagées qui sont liées à la manière de commander le robot. En commande manuelle, l'opérateur agit directement sur la base. En automatique, l'opérateur alloue une mission au robot qui se charge de la réaliser automatiquement. Entre ces deux extrêmes, des modes dits «partagés" permettent à l'opérateur de se concentrer sur un aspect précis de la mission: la détection et la gestion des collisions, la détection et la gestion des erreurs de localisation, la commande de la base mobile et la commande du bras manipulateur. Le robot se charge alors des éléments délaissés par la personne. Dans le cas de la commande automatique, le robot utilise des méthodes numériques de localisation et de navigation basées sur la vision.

Nous souhaitons, dans le travail présenté ici, trouver une solution à deux problèmes classiques de la robotique mobile : la localisation «fine» qui permet au robot de 
savoir à quelle distance il se trouve des objets de son environnement immédiat, et la localisation "grossière » qui a pour but de localiser le robot par rapport à une carte de son environnement sans rechercher une précision particulière. Pour illustrer cela, nous prenons un exemple. Pour accomplir une tache précise, le robot doit pouvoir calculer la distance aux éventuels obstacles présents sur son chemin et arriver à proximité de l'objet recherché avec précision. Nous sommes là dans un travail de localisation fine. Par contre si, à un moment donné dans l'accomplissement de sa tache, le robot ne sait plus où il est dans l'appartement de la personne, il doit pouvoir se resituer sommairement pour envoyer ses coordonnées à la base fixe (l'opérateur).

Le travail de localisation fine a été abordé sur des images en noir et blanc par Humberto Loaiza [3] : nous allons aborder ici les apports de la couleur à ce travail. Pour ce qui est de la localisation grossière (en vision monoculaire), des travaux ont déjà été réalisés au LSC par Omar Ait-Aider [4] sur des images en noir et blanc. L'intérêt de la couleur pour cette localisation grossière fait parti de nos centres d'intérêts futurs.

Loaiza a développé des méthodes de localisation et de navigation spécialement adaptées à un environnement domestique et basées sur une vision stéréoscopique monochromatique passive La notion d'environnement domestique est importante car l'espace, dans lequel opère la base mobile, est constitué majoritairement d'objets manufacturés qui sont par nature structurés (murs, plafonds, sols, portes, fenêtres, mobilier, électroménager) et qui ont des formes polyédriques et cylindriques. L'utilisation de primitives de type «segments de droite » est donc avantageuse. La notion de passivité implique l'absence d'aménagements spécifiques dans l'habitat et qui pourraient faciliter la localisation et la navigation du robot au détriment de la qualité de vie de la personne handicapée. Enfin, la notion de vision monochromatique est liée aux coûts passés des équipements de vision couleur qui étaient bien plus élevés que ceux de vision en noir et blanc. En outre, pour des raisons de simplicité, les recherches effectuées en traitements d'images sont couramment menées sur des sources unidimensionnelles, c'est à dire des images en niveaux de gris.

L'algorithme de construction des segments est basé sur le gradient de Deriche [5] et sur une segmentation par hystérésis. Le gradient de Deriche est simple et ne présente qu'un seul paramètre qui règle le compromis à accepter entre localisation et détection des éléments de l'image. Pour sa part, Loaiza a opté pour un compromis tel que les segments détectés sont principalement de grande taille. En effet, il est plus utile de se localiser grâce aux objets de grandes dimensions qui auront tendance à être peu souvent déplacés. Ensuite, par appariement des segments extraits de deux images stéréoscopiques, la représentation $3 \mathrm{D}$ de la scène est construite. La méthode d'appariement utilisée est une classification hybride (bayésienne et par réseau de neurones) de paires de segments en deux classes: «bien» ou «mal» appariés. Les arguments de la classification sont des descripteurs basés sur la géométrie du segment, la luminance et la texture des régions de part et d'autre du segment, et le voisinage avec les autres segments. Camille N'Zi a montré que cette méthode de classification est plus performante qu'une méthode épipolaire plus classique [6].

Dans l'optique d'une coopération homme-machine, il est préférable d'avoir un renvoi à l'opérateur d'images en couleur qui sont plus «naturelles» que des images monochromatiques. En conséquence, la base mobile a été pourvue d'une caméra couleur. Cependant, les algorithmes de localisation et de navigation implantés traitent des images en noir et blanc et n'exploitent donc pas l'information couleur plus complète. De plus, l'étude de nos habitats permet de constater la grande variété d'objets colorés et texturés qui les composent : parquets, tapis, carrelages, papiers-peints, peintures, meubles en bois ou modernes, objets en plastique, vitres, ... Par conséquent, il devient intéressant de se pencher sur l'exploitation possible des informations de couleur, mais aussi de texture, pour notre application. Dans un premier temps, notre but est d'étendre les méthodes de Loaiza à des images polychromatiques et d'évaluer cet apport. Un second objectif sera de trouver des objets colorés spécifiques et nécessaires à une tâche.

L'intégration de l'information couleur se heurte à deux problématiques majeures. Premièrement, il existe plusieurs manières de représenter les couleurs et un choix doit être fait. Deuxièmement, il faut passer d'une méthode monodimensionelle (niveaux de gris) à une méthode tridimensionelle (espace couleur 3D) de construction des segments. Dans cet article, nous présentons un rappel $\mathrm{du}$ processus original de segmentation ainsi que de la caractérisation des segments. Ensuite, nous introduisons la segmentation couleur que nous avons développée ainsi que la méthodologie utilisée pour évaluer l'importance de cet apport. Après la conclusion de ce travail, nous poursuivons sur les travaux actuellement en cours.

\section{SEGMENTATION NOIR ET BLANC}

\section{A. Extraction des segments}

Un environnement domestique se caractérise par un grand nombre d'objets structurés. Ces objets ont des formes polyédriques ou cylindriques pour la plupart car ils sont manufacturés. Les éléments importants d'une scène, à savoir la maçonnerie et le mobilier, présentent ainsi de grandes lignes verticales, horizontales et fuyantes, pour peu qu'ils ne soient pas masqués. Les autres objets présentent eux aussi des lignes mais dans une moindre mesure. De plus, l'utilisation de segments de droite permet la reconstruction 3D d'une scène. Ces considérations apportent à la segmentation, dans le sens extraction de segments, un fort intérêt. Nous allons maintenant décrire le processus développé initialement 
par Loaiza pour extraire des segments d'images stéréoscopiques.

Une telle prise d'images sous-entend l'emploi de deux caméras ayant des positions géométriques ou des orientations différentes. Le processus de segmentation s'effectue alors en parallèle sur les deux images acquises et comporte plusieurs étapes: correction des images, rehaussement des contours, seuillage des images enrichies, et extraction des segments à partir des contours.

La correction des images consiste en une correction des distorsions radiales et en un rehaussement $\mathrm{du}$ contraste (correction de dynamique).

Le rehaussement des contours se fait par une approche " gradient » qui permet de récupérer les angles aigus souvent présents dans les images d'intérieur. Le gradient est calculé par approximation en utilisant les filtres optimaux et séparables de lissage et de dérivation de Deriche [5]. Ce dernier, à partir du formalisme de Canny [11], a en effet proposé une solution basée sur un filtre à réponse infinie en modifiant les conditions initiales :

$$
f(x)=-c x e^{-\alpha|x|} \text { avec } c=\frac{\left(1-e^{-\alpha}\right)^{2}}{e^{-\alpha}}
$$

Ce filtre est plus performant que celui de Canny. De plus, l'opérateur de Deriche est assez simple et ne présente qu'un seul paramètre $\alpha$ qui règle le compromis entre les critères de localisation du robot et de détection des contours : si $\alpha$ diminue, la localisation diminue mais le rapport signal sur bruit augmente, soit une meilleure détection, et vice versa. Loaiza a fixé $\alpha$ à 1 . Pour améliorer l'immunité au bruit de l'image $I(x, y)$, un lissage est préalablement appliqué au calcul du gradient. Le filtre utilisé est l'intégrale du filtre optimal $f(x)$ :

$$
h(x)=k(\alpha|x|+1) e^{-\alpha|x|}
$$

Le calcul des composantes $I_{x}$ et $I_{y}$ du gradient se fait à partir des dérivées selon $x$ et $y$ du produit de convolution de l'image par le filtre de lissage. Compte tenu des règles de dérivation de l'opération de convolution et de la séparabilité des deux filtres, on a :

$$
\begin{aligned}
& I_{x}=[I(x, y) * h(y)] * f(x) \\
& I_{y}=[I(x, y) * h(x)] * f(y)
\end{aligned}
$$

La magnitude et l'angle du gradient sont calculés de manière conventionnelle à partir des deux composantes.

Le calcul du gradient, bien qu'essentiel, ne fournit cependant pas les points de contours. Une recherche de maxima locaux du gradient et le calcul de deux valeurs de seuillage haut et bas permettent d'obtenir, via un seuillage par hystérésis, des images de contours binaires " haut» et « bas ».

Une segmentation par hystérésis des deux images de contours, qui regroupe en parallèle l'étape de suivi de contours et la procédure de segmentation, fournit finalement une base de segments et leurs paramètres. Cette méthode est basée sur les critères de déviation de surface et d'écart entre pixels.
Les figures 1 et 2 présentent un résultat du traitement d'image et de l'extraction de segments sur deux images stéréoscopiques (dans cet exemple, la tête stéréo est verticale : caméras haute et basse).
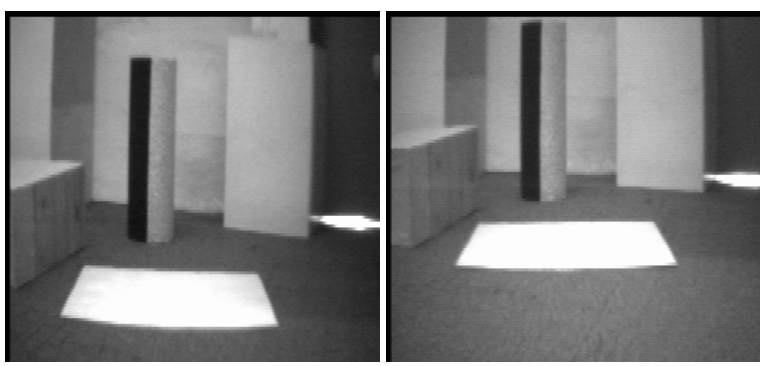

Fig.1. Images stéréoscopiques haute et basse

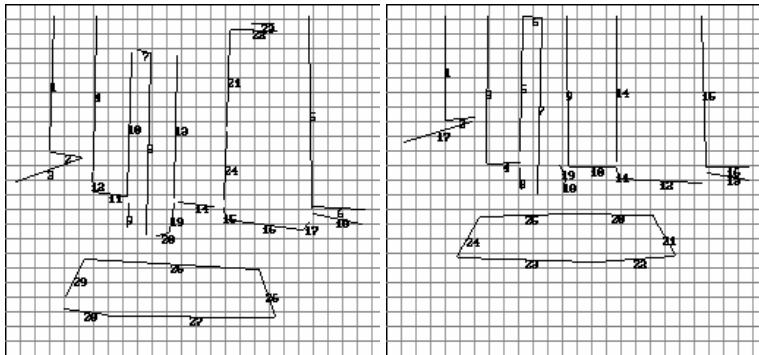

Fig.2. Images de la figure 1 après segmentation

\section{B. Caractérisation des segments}

Après avoir obtenu les segments, des paramètres sont calculés qui sont utilisés dans la phase d'appariement. Ces paramètres sont de quatre types: géométriques, de luminosité, de texture et de voisinage avec les autres segments. Certains sont calculés lors de la segmentation: coordonnées du milieu, longueur, orientation, norme moyenne du gradient. D'autres sont liés à la notion de régions à droite et à gauche des segments et sont obtenus à part : moyennes des niveaux de gris dans les deux régions, contrastes internes dans les deux régions, différences de niveaux de gris dans les deux régions suivant quatre directions élémentaires. Ces derniers paramètres sont issus d'une simplification de la matrice de cooccurrence. Deux paramètres de voisinage font appel à la technique des «buckets». En tout, 19 variables sont calculées (dont 17 de dimensions finies, ceux de voisinage sont des listes variables) Ce nombre est important d'autant plus que certains paramètres sont corrélés. Des méthodes basées sur l'analyse en composantes principales et l'analyse factorielle discriminante permettent de réduire ces paramètres à 8 .

\section{SEGMENTATION COULEUR}

Nous considérons trois approches différentes pour obtenir des segments tout en intégrant l'information couleur. Avant de les décrire, nous allons préciser quels sont les espaces couleur retenus dans notre travail. 


\section{A. Espaces couleur considérés}

Nous avons décidé de limiter le nombre d'espaces et avons choisi cinq espaces représentatifs de tous les autres. L'espace Rouge Vert Bleu est le plus commun. C'est l'espace par défaut des images «bitmap » à partir desquelles nous travaillons. L'espace xyz [7] est normalisé et donne le "diagramme chromatique ». L'espace $L^{*} a^{*} b^{*}[8]$ est un espace d'axes antagonistes qui tend à reproduire la perception des couleurs par le système visuel humain. $L^{*}$ est l'axe d'opposition entre le noir et le blanc, $a^{*}$ entre le vert et le rouge, $b^{*}$ entre le bleu et le jaune. L'espace Hue Saturation Intensity [9] est un espace intuitif qui considère la couleur comme se composant d'une teinte, d'une saturation et d'une intensité. Enfin, l'espace $\mathrm{I}_{1} \mathrm{I}_{2} \mathrm{I}_{3}$ [10] est basé sur une analyse en composantes principales avec $\mathrm{I}_{1}$ axe d'intensité, $\mathrm{I}_{2}$ axe d'opposition entre le rouge et le bleu, et $\mathrm{I}_{3}$ axe d'opposition entre le vert et les deux autres primaires.

\section{B. Approche 1 : segmentation marginale}

Cette approche est une reprise de la méthode initiale [3] développée pour des images en niveaux de gris (algorithme monodimensionnel). Les images de chaque axe couleur ( $i=1$ à 3 ) sont traités indépendamment les unes des autres et donnent trois ensembles de segments (un par axe couleur).

\section{Approche 2 : segmentation vectorielle}

Dans cette deuxième approche, nous allons construire les segments en tenant compte des trois axes couleur d'un même espace pour obtenir un unique groupe de segments. Pour ce faire, le gradient de Di Zenzo [12] est utilisé : il permet d'obtenir une magnitude et une orientation du gradient unique à partir de celles des trois composantes couleur d'une image.

\section{Approche 3 : " fusion » des contours}

Pour cette approche, nous conservons le processus jusqu'au point où six images binaires se côtoient (trois images de contours par rapport à un seuil haut et trois par rapport à un seuil bas). Nous créons alors une image unique "seuil haut» et une image unique "seuil bas" grâce à une addition logique. Le reste du processus n'est pas altéré.

\section{E. Exemples}

Les figures 3 à 6 présentent une image et un résultat de la segmentation suivant les trois approches. Notons que les segments affichés ont une taille minimale de 20 pixels afin d'alléger la représentation graphique. A travers ces exemples, nous pouvons remarquer qu'un espace ne donne pas systématiquement plus de segments qu'un axe. De même, la quantité et la taille des segments ne sont pas forcément dépendantes.

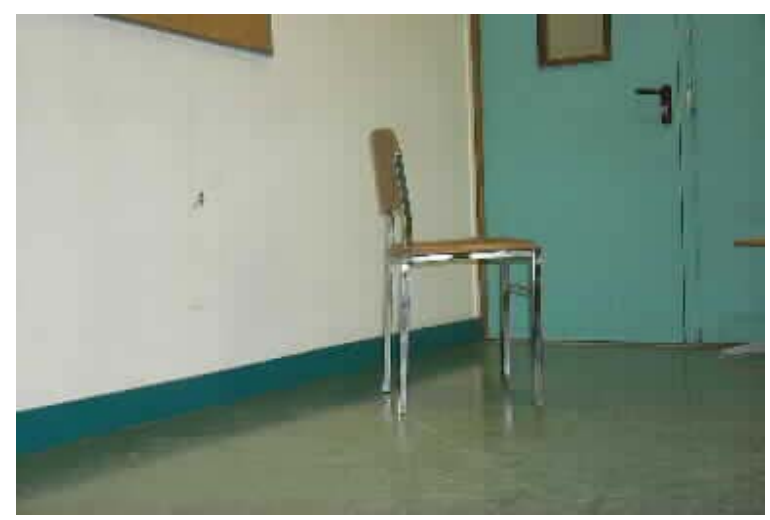

Fig.3. Une chaise

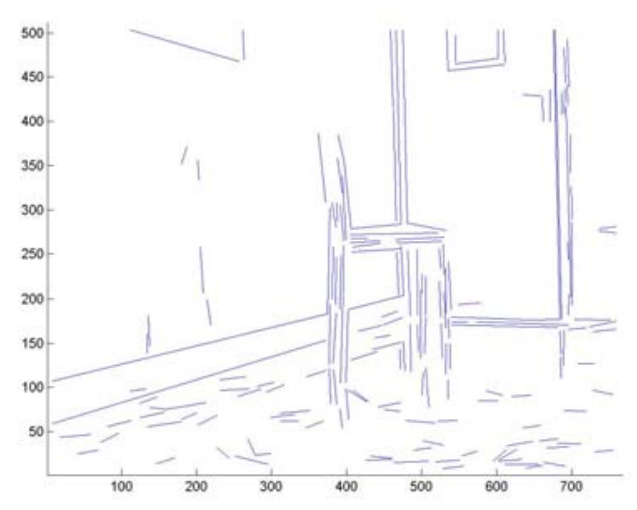

Fig.4. Segments issus de l'axe bleu (approche 1)

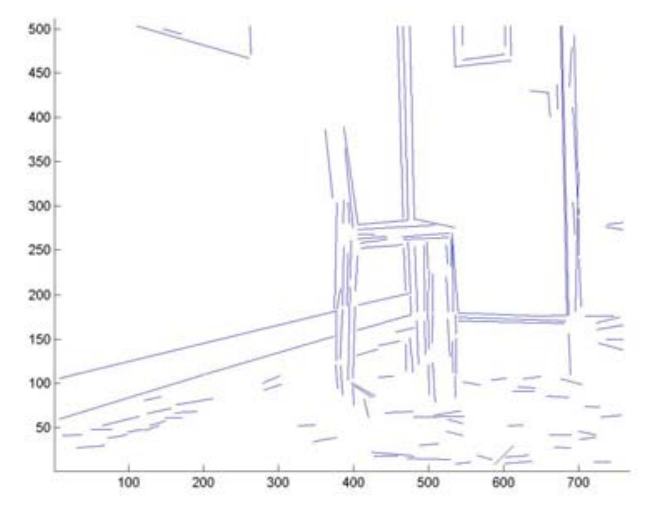

Fig.5. Segments issus de l'espace RVB (approche 2) 


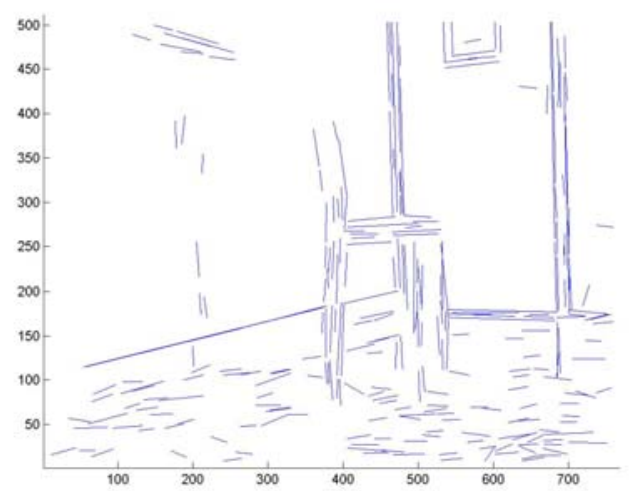

Fig.6. Segments issus de l'espace RVB (approche 3)

\section{F. Evaluation}

La qualité de la localisation et de la navigation dépend fortement de l'étape d'appariement. Et les résultats seront meilleurs si les segments extraits des images correspondent le plus possible aux éléments majeurs présents dans la scène.

Pour évaluer les espaces couleur, nous avons donc besoin d'outils de mesure fournissant des données de comparaison utiles. Peu de travaux ayant été fait à ce propos (exception faite de travaux spécifiques que nous ne pouvons reprendre directement [13][14]), nous avons développé nos propres outils afin qu'ils accordent plus d'importance aux segments de grande taille [15]. En effet, ces segments correspondent à un élément significatif de la scène et seront probablement détectés sur les deux images, prises par le système de vision stéréoscopique, conduisant ainsi à un appariement favorable. De plus, il est possible d'oublier certains éléments de petites dimensions: feuillage des plantes, carreaux au sol, motifs texturaux, et objets décoratifs.

Après avoir appliqué les algorithmes de segmentation aux différentes images d'axes ou d'espaces couleur, nous analysons les segments détectés avec des outils statistiques classiques tels la quantité de segments, la taille moyenne et l'écart-type (sur tous les segments ou sur ceux ayant une taille minimale). Nous utilisons aussi un système de notation qui peut être absolu (« notation pondérée ») ou relatif ( « comparaison d'histogrammes des segments ») et qui est décrit ci-dessous.

\section{1) Notation pondérée}

Nous distribuons les segments construits sur une échelle de taille dont le pas (en pixels) est fixé arbitrairement. Puis, pour chaque pas de taille, nous calculons une note en multipliant l'indice de pas $i$ par la quantité $q$ de segments correspondant. La note finale (éq. 1) est la somme de toutes les notes ainsi calculées.

$$
\text { note }=\sum_{i=0}^{n}\left(i \cdot q_{i}\right)
$$

Cette opération favorise les segments de grande taille. Ainsi, l'axe ou l'espace couleur qui fournit le plus grand nombre de grands segments obtiendra la meilleure note et sera considéré comme étant le plus performant. Ayant choisi cinq espaces couleur (RVB, xyz, HSI, $\mathrm{L} * \mathrm{a}^{*} \mathrm{~b}, \mathrm{I}_{1} \mathrm{I}_{2} \mathrm{I}_{3}$ ), nous avons donc quinze axes couleur. A toutes les images de notre base, nous appliquons notre méthode et comparons les notes obtenues afin de sélectionner les axes ou l'espace conduisant aux meilleurs résultats. Dans notre cas, le pas de taille choisi est égal à 25 pixels. Ainsi les segments qui ont une taille inférieure à 25 pixels ne contribuent pas à la notation.

\section{2) Comparaison d'histogrammes de segments}

Afin d'évaluer la qualité des images colorées par rapport aux images monochromes, nous comparons l'histogramme des segments d'une image avec celui de la même image en niveaux de gris. Nous considérons, pour l'image en niveaux de gris, la moyenne des trois composantes de l'espace RVB, ce qui correspond à l'axe $\mathrm{I}_{1}$. A partir de ces deux histogrammes, nous calculons une autre note, comme expliqué ci-dessous :

$$
\begin{gathered}
\text { note }=\sum_{i=0}^{n}\left(i \cdot \Delta q_{i}\right) \\
\Delta q_{i}=\sum_{j=\text { bas }_{i}}^{j=\text { haut }_{i}}\left(q-q_{r}\right)_{j}
\end{gathered}
$$

Où $i$ est l'indice de pas, $q$ la quantité de segments issus de l'image couleur, et $q_{r}$ la quantité de segments issus de l'image de référence en niveaux de gris. Pour un pas donné $i$, nous avons une longueur minimale $\left(b a s_{i}\right)$ et une longueur maximale (haut $)_{i}$ ) de segment.

Notons que cette dernière approche inclut la notation pondérée tout en apportant la possibilité de comparer chaque axe couleur avec une même référence.

\section{3) Résultats}

Nous avons une base de plus de 300 images couleur de différents intérieurs. Ces images sont aussi bien des photographies prises par nos soins que des photographies issues de bases déjà existantes. De là, nous avons recueilli différentes données mais seules trois d'entre-elles sont significatives: quantité de segments, taille moyenne des segments, et comparaison d'histogrammes de segments.

En comparant les axes les uns avec les autres comme le permet l'emploi de la première méthode de segmentation, nous avons constaté de bons résultats sur les axes d'intensité $\mathrm{L}^{*}$, I et $\mathrm{I}_{1}$ (qui est aussi l'axe des images en niveaux de gris), ainsi que sur les axes contenant en partie une information d'intensité R, $\mathrm{V}$ et $\mathrm{B}$. Cependant seul $\mathrm{L}^{*}$ donne de meilleurs résultats que notre référence $\mathrm{I}_{1}$. Cela veut dire que les axes couleur étudiés donnent moins de segments, à longueurs équivalentes, que l'axe d'intensité (images en niveaux de gris). Ce constat n'est pas un non-sens car les axes couleur portent le plus souvent une information réduite par rapport à l'intensité lumineuse. 
En comparant non plus les axes mais les espaces couleur (par l'emploi des méthodes 2 et 3 et en moyennant les résultats des axes d'un même espace par la méthode 1), nous constatons que l'axe d'intensité reste plus performant quelle que soit la méthode employée. Outre le fait que certains espaces soient par nature peu adaptés (xyz notamment), la détérioration de l'information d'intensité par les deux autres axes (HSI, $\mathrm{I}_{1} \mathrm{I}_{2} \mathrm{I}_{3}, \mathrm{~L}^{*} \mathrm{a}^{*} \mathrm{~b}^{*}$ ) ou la distribution de cette information sur les trois axes (RVB) expliquent ces résultats.

Par ailleurs, l'approche 2 basée sur le gradient de Di Zenzo donne moins de segments que l'approche 3 basée sur la « fusion des contours » mais ces segments sont plus longs. En effet, la $2^{\text {ème }}$ approche est basée sur un gradient unique mais optimisé qui donne des segments collant au plus juste les éléments d'une scène. L'approche 3, par contre, donne des contours plus complets à l'algorithme de segmentation avec, pour conséquence, la création de nombreux segments correspondent aux détails de la scène.

Pour conclure, nous pouvons affirmer que l'emploi d'images en niveaux de gris est suffisant et efficace pour l'extraction de segments dans une image. Cependant, il est possible d'utiliser la couleur avantageusement. En effet, si les segments créés à partir de chaque axe couleur d'un même espace sont mis en commun, un ensemble unique de segments est alors créé (emploi dérivé de la $1^{\text {ère }}$ méthode de segmentation). De manière logique, les résultats sont meilleurs puisqu'ils consistent à sommer les résultats de trois segmentations indépendantes, et cela d'autant plus si un des axes est un axe d'intensité. Cette dernière approche génère des segments redondants mais aussi des segments complémentaires.

\section{G. Caractérisation des segments dans un espace couleur}

Les paramètres que nous avons présentés dans le paragraphe 2.2 sont de différentes natures : géométriques, de luminance, de texture et de voisinage. Certains de ces paramètres (comme ceux de géométrie et de voisinage) sont réutilisables dans un espace couleur. Il n'en va pas de même pour les paramètres de luminance et de texture.

Le problème auquel nous sommes confrontés est de répondre aux questions suivantes: "Quels sont les paramètres caractéristiques d'un environnement couleur? Est-ce que la texture peut-être abordée de la même façon dans une image couleur que dans une image noir et blanc ? Est-ce qu'il faut retenir les mêmes paramètres sur des images présentant des caractéristiques de couleur très différentes ? » Pour illustrer ces questions, nous allons nous appuyer sur deux images extraites de notre base de donnée. Celle de la figure 7 montre un environnement peu homogène et présente des couleurs très vives, contrairement à celle de la figure 8 . On constate que, si sur la $1^{\text {ère }}$ image les contours séparent souvent des régions de couleurs et de texture très différentes, il n'en va pas de même pour l'autre image où la teinte bois / marron est dominante : dans la $2^{\text {ème }}$ image, les segments extraits sépareront des zones d'une même région colorimétrique.

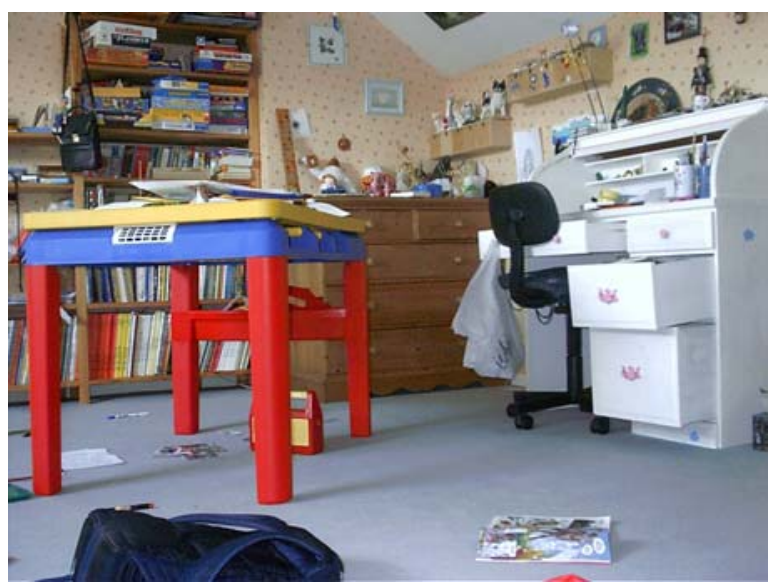

Fig.7. Image peu homogène

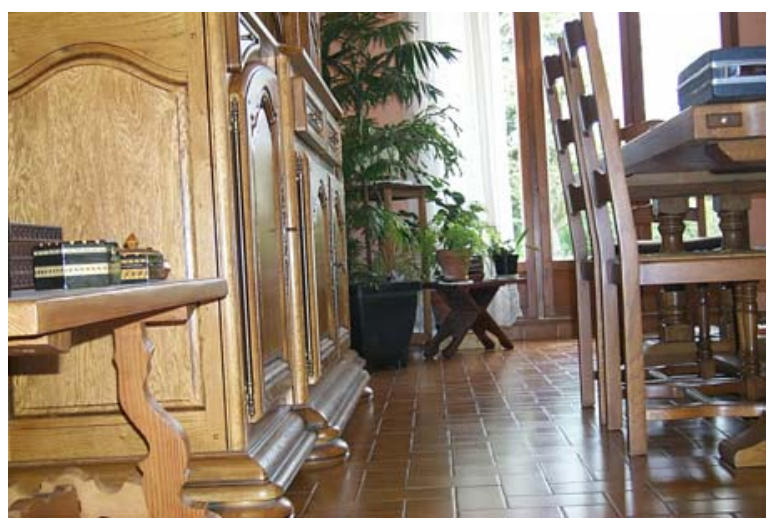

Fig.8. Image homogène

Nous avons donc réfléchi à la mise en œuvre d'un système adaptatif permettant de choisir automatiquement l'axe colorimétrique le plus discriminant. Axe sur lequel nous viendrons calculer les paramètres caractéristiques des régions encadrant le segment. Pour cela, nous travaillons suivant deux directions différentes. La $1^{\text {ère }}$ voie de recherche consiste à projeter toutes les images de la base sur un espace couleur extrêmement réduit (palette fixe de 256 couleurs) comme le montre les figures 9 et 10 .

Cet espace est visuellement décevant mais il nous permet de pouvoir comparer les histogrammes (voir figures 11 et 12) de toutes les images de notre base dans un référentiel unique. L'analyse de ces histogrammes permet d'obtenir rapidement une information sur les caractéristiques de l'image par différents calculs statistiques et l'étude des maxima: couleur prédominante, contraste, dynamique. 


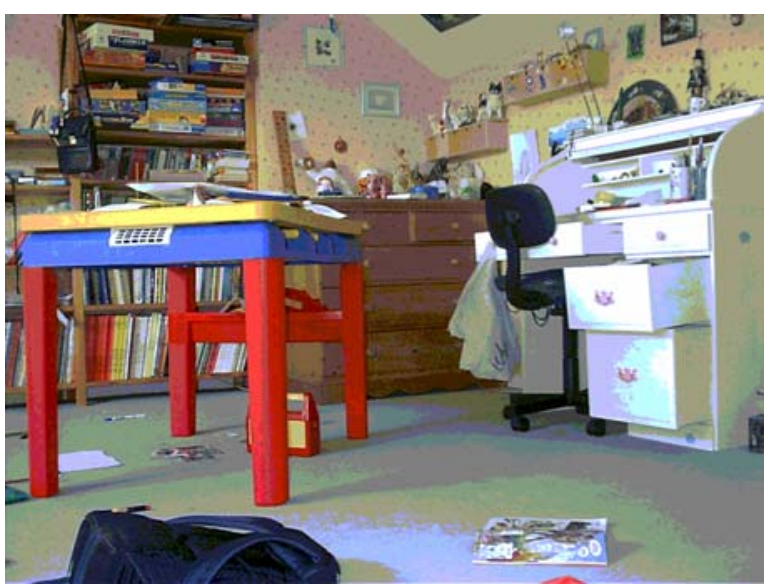

Fig.9. Image peu homogène réduite à 256 couleurs (palette fixe)

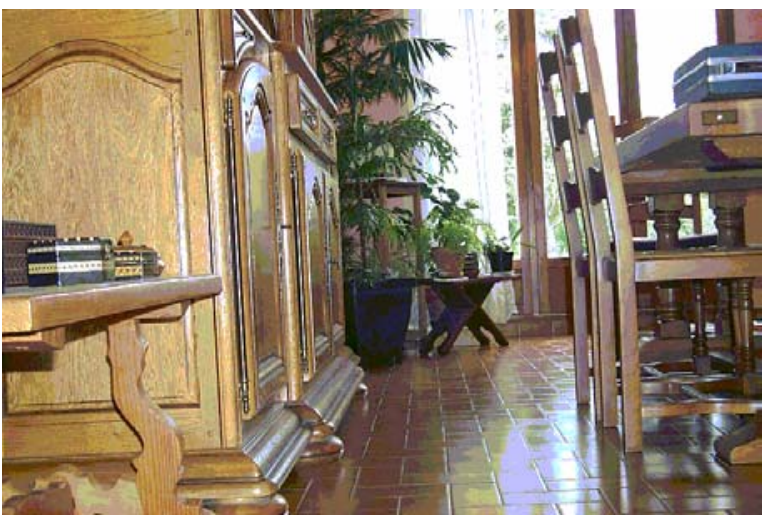

Fig.10. Image homogène réduite à 256 couleurs (palette fixe)

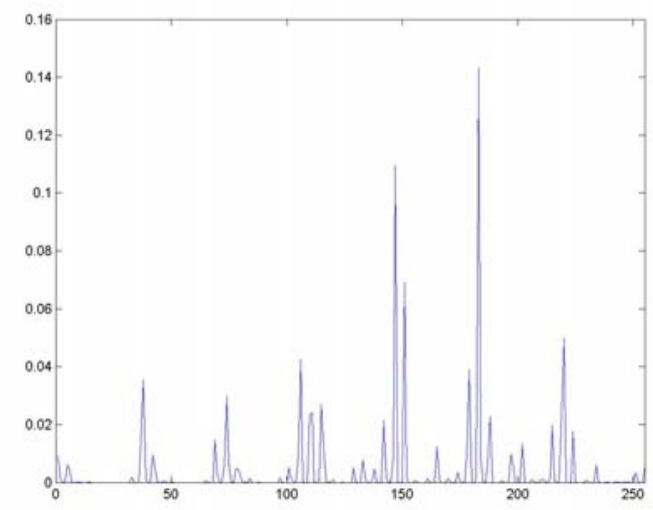

Fig.11. Histogramme de la figure 9

La $2^{\text {ème }}$ voie de recherche consiste à extraire une sous-image en sous-échantillonnant l'image d'origine comme le montre la figure 13. Pour cela nous utilisons les résultats présentés par André Smolarz [16].

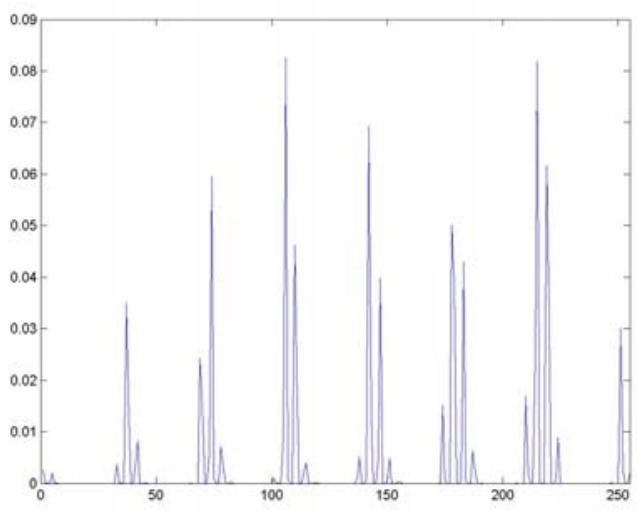

Fig.12. Histogramme de la figure 10

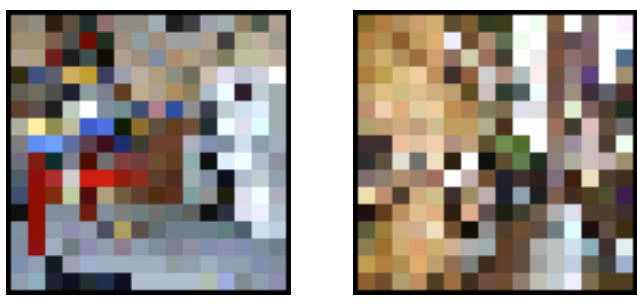

Fig.13. Sous-images $16 \times 16$

En comparant les paramètres statistiques des images et des « imagettes", nous voyons que ces dernières, constituées de 256 pixels, proposent une représentation fiable des images d'origine.

A partir de paramètres calculés sur les histogrammes et les sous-images, il paraît possible de faire le choix de l'axe couleur le plus pertinent pour le calcul des caractéristiques des régions encadrant le segment à apparier. Pour étayer ce principe nous avons constitué des classes d'images en nous appuyant sur une mesure de différentes distances inter-histogrammes, notamment les distances de Kullback-Leibler [17] et de Minkowsi [18]. Ces classes mettent en évidence une homogénéité de teinte parmi les images constituant chaque classe. Il nous faut maintenant déterminer l'axe couleur le plus adapté à chacune de ces classes. Les paramètres moyens des «imagettes" et des histogrammes de chacune de ces classes pourraient servir de mesures de comparaison avec les paramètres calculés sur l'image acquise par le robot, nous permettant ainsi de choisir l'axe le plus adapté pour caractériser des segments.

\section{CONCLUSION}

En l'état, il est possible de dire que les axes d'intensité tels $\mathrm{L}^{*}$, I ou $\mathrm{I}_{1}$ sont, de manière individuelle, bon pour obtenir suffisamment de segments dans le cadre d'un processus de localisation et de navigation d'un robot. Comme un axe couleur a moins d'information qu'un axe d'intensité, il est inutile de l'utiliser seul. Cependant, l'information de couleur peut compléter celle d'intensité dans certains cas. De plus, il est toujours 
possible d'utiliser avantageusement l'information couleur en utilisant de multiples ensembles de segments issus des trois axes d'un même espace. Dans ce cas, nous devons opérer une sélection des segments pour limiter le phénomène de redondance lié à cette méthodologie. Nous pouvons aussi envisager de rechercher un espace de représentation original ou introduire une sélection automatique (ou, à défaut, une non-sélection) d'axes/espaces, au moyen d'autres outils. Ces outils pourront être inspirés de travaux sur l'indexation et la recherche d'images [16][19].

$\mathrm{Au}$ final, nous devons expérimenter l'appariement des segments pour conclure objectivement. Dans ce cas, en plus de la méthode existante, une seconde méthode sera utilisée basée sur la géométrie épipolaire [5] qui permettra de comparer les deux approches et éventuellement de compléter les résultats fournis par la méthode initiale.

\section{REFERENCES}

[1] P. HOPPENOT, «Contribution de la robotique mobile à l'assistance aux personnes handicapées », 27 novembre 1997, Thèse de Doctorat, Université d'Evry.

[2] P. Hoppenot, E. Colle, O. Ait Aider, Y. Rybarczyk, «ARPH - Assistant Robot for Handicapped People - A pluridisciplinary project », IEEE Roman'2001, Bordeaux et Paris, France, 2001, pp. 624-629.

[3] H. Loaiza, J. Triboulet, S. Lelandais, C. Barat, "Matching segments in stereoscopic vision», IEEE Instrumentation \& Measurement Magazine, vol.4, $\mathrm{n}^{\circ} 1$, Mars 2001, pp.37-42.

[4] O. Ait-Aider, "Localisation référencée modèle d'un robot mobile d'intérieur par vision monoculaire», 19 décembre 2002, Thèse de Doctorat, Université d'Evry.

[5] R. Deriche, "Using Canny's criteria to derive an optimal edge detector recursively implemented », The International Journal of Computer Vision, 1987, pp.167187.

[6] C. N'Zi, H. Loaiza, J. Triboulet, S. Lelandais, C. Barat, C. Montagne, «Matching stereoscopic segments: comparison between epipolar, bayesian and neuronal approaches ", International Symposium on Measurements and Control in Robotics, session B8, Bourges, France, 2002.

[7] Publié sous la direction de Murat Kunt, «Traitement numérique des images », Traitement de l'information: volume 2, Collection Electricité, Presses Polytechniques et Universitaires Romandes, 1993.

[8] Alain Tremeau, "Représentation numérique des images couleur », Ecole de printemps - Images couleur, Pau, France, Mars 2001.

[9] Rafael C. Gonzales, Richard E. Woods, « Digital Image Processing ", Addison-Wesley Publishing Co., Massachusetts, 1993, pp.221-251.

[10] Yu-Ichi Ohta, Takeo Kanade, Toshiyuki Sakai, " Color information for region segmentation », Computer Graphics and Image Processing 13, 1980, pp.222-241.
[11] J. F. Canny, «A computational approach to edge detection », IEEE Transactions on Pattern Analysis and Machine Intelligence, vol.8, pp.679-698, 1986.

[12] Silvano Di Zenzo, «A note on the gradient of a multi-image », Computer Vison, Graphics and Image Processing, vol.33, 1986, pp.116-125.

[13] Jean-Christophe Terrillon, Yoshinori Niwa, Kazuhiko Yamamoto, «On the selection of an efficient chrominance space for skin color-based image segmentation with an application to face detection ", QCAV 2001, Le Creusot, France, Mai 2001, pp.409-414.

[14] Alberto Albiol, Luis Torres, Edward J. Delp, "Optimum color spaces for skin detection", International Conference on Image Processing, Octobre 2001, pp.122-124.

[15] C. Montagne, S. Lelandais, J. Triboulet, C. N'ZI, « How to choose the best color space for the guidance of an indoor robot?», CGIV 2002 - The First European Conference on Colour in Graphics, Image and Vision, Poitiers, France, Avril 2-5, 2002, pp. 589-593.

[16] A. Somlarz, P. Cornu, « A color image classification by means of image transformations », CGIV 2002 - The First European Conference on Colour in Graphics, Image and Vision, Poitiers, France, Avril 2-5, 2002, pp. 310-315.

[17] S. Kullback, R.A. Leibler, " On information and sufficiency », Annals of Mathematical Statistics, vol.22, pp.79-86, 1951.

[18] D. Peeters, I. Thomas, « Distance- $\mathrm{L}_{\mathrm{p}}$ et localisations optimales. Simulations sur un semis aléatoire de points », Cahiers Scientifiques du Transport, 1997, vol.31, pp.5570.

[19] Stuart Gibson, Richard Harvey, J. Andrew Bangham, « Multi-dimensional histogram comparison via scale trees ", International Conference on Image Processing, Thessaloniki, Grèce, Octobre 2001, pp.709712. 